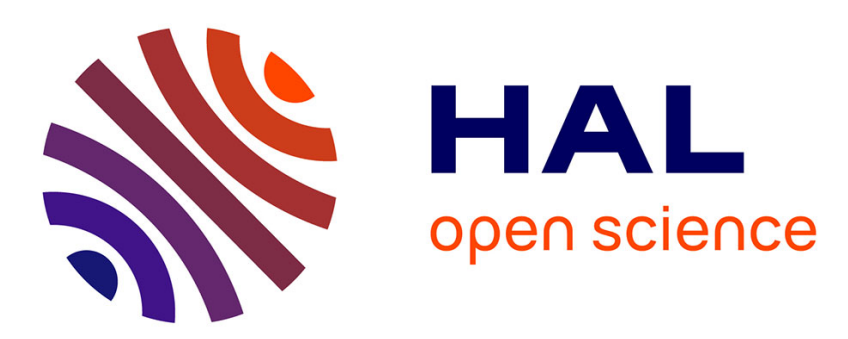

\title{
Le chant prophétique dans les Dialogues des carmélites. De la représentation à la réactualisation du martyre
}

\author{
Maud Pouradier
}

\section{To cite this version:}

Maud Pouradier. Le chant prophétique dans les Dialogues des carmélites. De la représentation à la réactualisation du martyre. Transversalités, 2020, Poésie et transcendance, 4 (155), pp.101-116. 10.3917/trans.155.0101. hal-02874894

\section{HAL Id: hal-02874894 https://hal.science/hal-02874894}

Submitted on 19 Jun 2020

HAL is a multi-disciplinary open access archive for the deposit and dissemination of scientific research documents, whether they are published or not. The documents may come from teaching and research institutions in France or abroad, or from public or private research centers.
L'archive ouverte pluridisciplinaire HAL, est destinée au dépôt et à la diffusion de documents scientifiques de niveau recherche, publiés ou non, émanant des établissements d'enseignement et de recherche français ou étrangers, des laboratoires publics ou privés. 


\title{
Le chant prophétique dans les Dialogues des carmélites. De la représentation à la réactualisation du martyre.
}

\author{
Maud Pouradier \\ Normandie Univ, UNICAEN, Identité et subjectivité, 14000 Caen, France
}

Dialogues des carmélites fait partie de ces opéras qui, comme Pelléas et Mélisande de Debussy, firent presque oublier l'œuvre originelle dont ils étaient inspirés. Bernanos écrivit en 1948 des dialogues pour un film consacré au martyre des bienheureuses carmélites de Compiègne en 1794. Après la mort de l'auteur, le texte fut remanié en pièce de théâtre par Albert Béguin et Marcelle Tassencourt, avant qu'il ne devienne le livret de l'opéra de Poulenc créé en 1957. Le texte de Bernanos finit par donner lieu à un film en 1960 : Le dialogue des carmélites, réalisé par Philippe Agostini et Raymond Bruckberger. De toutes ces réalisations génériques différentes, c'est l'opéra de Poulenc qui a conféré à l'œuvre de Bernanos sa postérité. Cette réussite n'est pas seulement musicale, mais fictionnelle. L'histoire du personnage fictif de Blanche de la Force, inséré dans le récit du martyre des carmélites sous la Terreur, est mieux racontée par l'opéra que par le théâtre ou le cinéma, et sa puissance émotionnelle sur le spectateur est plus forte que dans les autres médiums. Dialogues des carmélites ont-ils une spécificité expliquant que sa meilleure réalisation générique fût un opéra? On a assez souvent raconté des événements historiques et religieux à l'opéra : outre les opéras bibliques comme Nabucco de Verdi ou Samson et Dalila de Saint-Saëns, on peut penser aux Huguenots de Meyerbeer ou à Giovanna d'Arco de Verdi. L'originalité du livret des Dialogues des carmélites réside dans la place qu'y occupe la prophétie. Au premier acte, la prieure des carmélites de Compiègne meurt en ayant la vision d'une chapelle « vide et profanée » avec du « sang sur les dalles ». Vient d'être acceptée comme novice Blanche de la Force, jeune aristocrate habitée par une foi vive, mais souffrant d'une peur existentielle mystérieuse sans objet clair - l'angoisse. Sœur Constance est le double de sœur Blanche : également novice et aristocrate, elle est au contraire habitée par une joie invincible qui frise parfois la légèreté. Elle semble par ailleurs avoir don de prophétie : elle prédit à sœur Blanche que le jour de sa mort, elle sera libérée de sa peur par la grâce de la mort de la prieure, qui aurait échangé son agonie contre celle de Blanche. Ces deux prophéties se réalisent au dernier acte de l'opéra : les carmélites sont condamnées à mort pour avoir mené des "conciliabules contre-révolutionnaires » et pour avoir conservé des "écrits liberticides». Conformément au vœu du martyre qu'elles ont prononcé contre l'avis de madame Lidoine, leur nouvelle prieure issue de la bourgeoisie, les religieuses sont menées à l'échafaud tandis qu'elles chantent Salve Regina. Alors qu'elle s'était enfuie, sœur Blanche reçoit miraculeusement le don de la force à laquelle son nom la prédestinait. Elle rejoint ses compagnes pour accueillir la grâce du martyre, sous le regard de Mère Marie de l'Incarnation, personnage historique de qui nous tenons le récit du martyre effectif des carmélites de Compiègne. Comme l'explique Joseph Ratzinger, la prophétie authentique n'est pas une simple prédiction de l'avenir :

[La] prophétie, au sens biblique, ne signifie pas prédire l'avenir, mais expliquer la volonté de Dieu pour le présent, et donc montrer la voie droite vers l'avenir. Celui qui prédit l'avenir satisfait à la curiosité de la raison, qui désire ouvrir le voile de l'avenir; le 
prophète, quant à lui, satisfait à l'aveuglement de la volonté et de la pensée, et éclaire la volonté de Dieu comme exigence et indication pour le présent ${ }^{1}$.

Comment intégrer une prophétie dans un récit tout en conservant son sens biblique ? En tant qu'elle fait partie du système de faits de l'histoire, elle doit trouver son aboutissement au terme du récit - au risque de la transformer en simple prédiction. En tant qu'elle prétend être une prophétie authentiquement chrétienne, elle doit conserver son actualité pour l'auditeur de tous les temps et avoir une dimension eschatologique au risque de l'éclatement du récit. Comment la prophétie peut-elle avoir un statut intradiégatique tout en conservant son actualité pour le spectateur? Comment peut-elle clore le récit et l'ouvrir? Comment la prophétie peut-elle à la fois être représentée dans la distance de la fiction, et s'incarner effectivement pour le public ? C'est cette gageure que l'opéra de Poulenc réussit mieux que les autres médiums qui s'y essayèrent. Dans cette réussite, quelle est la part du genre de l'opéra en général, et du traitement musical de Poulenc en particulier? Après une brève étude de la manière dont les coupes effectuées par Poulenc sur le texte de Bernanos en modifient le sens, on réfléchira à la manière dont le dispositif opératique construit une énonciation prophétique valide à l'intérieur et à l'extérieur du récit. On s'attachera ensuite à la manière dont Poulenc met en place une énonciation musicale prophétique avec la figure mélodique « de la mort», extraite du Stabat mater de 1951, pour construire la scène finale comme une réactualisation quasi-eucharistique du martyre des carmélites de Compiègne.

\section{Des dialogues de Bernanos aux Dialogues de Poulenc}

Avant d'élucider le rôle du traitement musical, il est nécessaire de prendre la mesure des modifications entraînées par le passage du texte de Bernanos au livret de Poulenc. Aucune comparaison systématique n'a été faite entre le livret et le texte édité par Albert Béguin. Quand celle-ci est esquissée, seul le gain en efficacité dramatique est mentionné ${ }^{2}$. Or le sens même du texte bernanosien est profondément modifié par Poulenc. En termes quantitatifs, le compositeur réduit de moitié le matériau textuel à sa disposition $^{3}$. Il vit deux fois le spectacle donné au théâtre Hébertot ${ }^{4}$, selon un texte adapté par Marcelle Tassencourt ${ }^{5}$, qui avait resserré l'action : la scène de la prison avec le marquis de la Force n'avait déjà pas été montée sur scène. Béguin et Tassencourt

\footnotetext{
${ }^{1}$ Joseph RATZINGER, « Le message de Fatima », publié le 26 juin 2000 par la Congrégation pour la doctrine de la foi [En ligne],

https://www.vatican.va/roman_curia/congregations/cfaith/documents/rc_con_cfaith_doc_20000626_mess age-fatima_fr.html [page consultée le 3 mai 2020].

${ }^{2}$ Denis WALECKX, "In search of a libretto», Francis Poulenc, music, art and literature, Sidney BuCKLAND et Myriam CHIMÈNES (éd.), Ashgate, 1999, p. 267.

${ }^{3}$ Il conserve 825 des 2618 lignes du texte bernanosien. Claude GENDRE, « Genèse de l'opéra de Francis Poulenc Dialogues des carmélites », Études bernanosiennes, n²2, 2001, p. 228.

${ }^{4}$ Denis WALECKX, «In search of a libretto », op. cit., p. 252.

${ }^{5}$ Dialogues des carmélites, texte de Georges Bernanos adapté par Albert Béguin et Marcelle Tassencourt, L'Avant-Scène Théâtre, $\mathrm{n}^{\circ} 337$, juillet 1965, p. 10-36. Il s'agit de la première édition du texte adapté pour le théâtre Hébertot en 1953. Poulenc n'a évidemment à sa disposition que l'édition d'Albert Béguin de 1949. Le compositeur ayant toutefois conservé toutes les coupes de la pièce de théâtre, on peut supposer que, sans avoir eu en main le texte adapté par Albert Béguin et Marcelle Tassencourt, le souvenir précis des représentations auxquelles il a assisté a influencé les choix qu'il fit pour le livret.
} 
avaient ajouté des phrases « utilitaires ${ }^{6} »$, que Poulenc conserva partiellement, notamment la gardienne criant « Blanche, il est temps de préparer les légumes ${ }^{7}$.»

Aux suppressions du théâtre Hébertot, Poulenc ${ }^{8}$ ajouta un réagencement complet des événements du texte bernanosien pour composer le second acte, en conservant parfois la phrase d'une scène supprimée pour la réintégrer dans la bouche d'un autre personnage (c'est notamment le cas de la phrase, au jardin des oliviers, le Christ n'était plus maître de rien, il a eu peur de la mort, prononcée dans l'opéra à la Conciergerie par madame Lidoine, et qui synthétise une réplique de sœur Marthe et une réplique de sœur Claire à la scène IX du quatrième tableau). Quant aux apports extrinsèques aux dialogues de Bernanos, il faut ajouter, à la phrase sur l'épluchage des légumes, la scène de l'acte de condamnation, probablement inspirée de la pièce de théâtre.

Acte III, $3^{\mathrm{e}}$ tableau.

Le Tribunal révolutionnaire expose que les ex-religieuses carmélites, demeurant à Compiègne, département de l'Oise, Madeleine Lidoine [Marie Madeleine Claudine Lidoine], Anne Pellerat [Marie Annette Pelras], Madeleine Touret [Anne Marie Madeleine Thouret], Marie-Anne Hanniset [idem], Marie-Anne Piedcourt [idem], MarieAnne Brideau [Marie Anne Françoise Brideau], Marie-Cyprienne Brare [Marie-Claude Cyprienne Brard], Rose Chrétien [Rose Crétien de Neuville], Marie Dufour [idem], Angélique Roussel [idem], Marie-Gabrielle Trézelle [Marie Gabrielle Trezel], MarieGeneviève Meunier [idem], Catherine Soiron [Anne Catherine Soiron], Thérèse Soiron [idem], Élisabeth Vézolot [Élisabeth Juliette Vérolot], ont formé des rassemblements et conciliabules contre-révolutionnaires, entretenu des correspondances fanatiques, conservé des écrits liberticides. Ne forment qu'une réunion de rebelles, de séditieuses qui nourrissent dans leurs cœurs le désir et l'espoir criminel de voir le peuple français remis aux fers de ses tyrans et la liberté engloutie dans des flots de sang que leurs infâmes machinations ont fait répandre au nom du ciel. Le Tribunal révolutionnaire déclare en conséquence que toutes les prévenues susnommées sont condamnées à mort ${ }^{9}$.

Le texte de la scène dans l'adaptation théâtrale de Béguin et Tassencourt, que Poulenc vit deux fois, était le suivant :

Tableau 19, scène 2, de l'adaptation Béguin-Tassencourt

Citoyenne Rose Chrétien de la Neuville, ci-devant Sœur Valentine. (À chaque nom, la religieuse qui le porte se lève doucement, en place). Citoyenne Lidoine, dite Marie de Saint-Augustin, se prétendant Prieure dudit Monastère. Citoyenne Marie-Jeanne de Suberville, ci-devant Sœur Constance de Saint-Denis. Citoyenne Marie-Anne Brideau, cidevant Sœur Mathilde. Citoyenne Madeleine Thouret, ci-devant Mère Jeanne de l'EnfantJésus. Citoyenne Catherine-Charlotte Brard, ci-devant Sœur Anne de la Croix. Citoyenne Marie-Gabriel Trezel, ci-devant Sœur Saint-Charles. Citoyenne Pelras, ci-devant Sœur Antoine. (Toutes les religieuses sont debout dans la demi-obscurité. Immobiles.) Citoyenne Françoise-Geneviève de Noyon, ci-devant Mère Marie de l'Incarnation, jugée par contumace. Citoyenne Blanche de la Force, également en fuite. Les dites personnes,

\footnotetext{
${ }^{6}$ Marcelle TASSENCOURT, avant-propos à L'Avant-Scène Théâtre, $\mathrm{n}^{\circ} 337$, juillet 1965, p. 7.

${ }^{7}$ Ibid., p. 8 .

${ }^{8}$ Denise Duval lui suggéra plusieurs coupes. Denis WALECKX, «Poulenc et ses interprètes », Alban RAMAUT (éd.), Francis Poulenc et la voix. Texte et contexte, Lyon/Saint-Etienne, Symétrie/Presses de l'université de Saint-Etienne, 2002, p. 1-10.

${ }^{9} \mathrm{~J}$ 'ai inséré entre crochets dans le texte du livret les noms des véritables carmélites de Compiègne. Source : SEEUR MARIE DE L'INCARNATION [Françoise Geneviève Philippe], La Relation du martyre des seize carmélites de Compiègne. Manuscrits inédits, William BusH (éd.), Paris, Cerf, 1993, p. 289-292.
} 
attendu qu'elles ont formé des conciliabules contre-révolutionnaires, entretenu des correspondances fanatiques et conservé des écrits liberticides, en vertu des décrets de la République, sont condamnées à mourir sur l'échafaud et seront remises sur-le-champ aux officiers chargés de l'exécution de la présente sentence. (Ils sortent)

Poulenc ne conserva que les noms civils : la répétition du ci-devant était contraignante, et la liste trop longue à traiter musicalement. La perte d'information pour le spectateur est compensée par l'effet de réel produit par ce martyrologe déguisé. Poulenc prend ainsi soin de respecter les véritables noms, qu'il ne modifie que pour des raisons musicales (ajout d'un $e$ à Trézel) ou poétiques (transformation de Vérolot en Vézolot). Le compositeur fait plusieurs autres coupes dans le texte bernanosien. La suspension des vœux religieux par l'Assemblée nationale n'est plus évoquée. Le long échange entre mère Marie et les commissaires du troisième tableau (scènes X et XI) n'apparaît plus. Poulenc supprime toutes les récréations, où les carmélites évoquent les bouleversements sociaux en cours, et où apparaissent les tensions sociales (troisième tableau, scène VI). Les Dialogues selon Poulenc taisent ainsi tous les enjeux sociaux et éthiques du texte de Bernanos, pour ne conserver que le drame spirituel et psychologique de la peur, de l'angoisse, et de la grâce ${ }^{10}$. Les références à l'honneur dans la bouche de Blanche de la Force perdent, dans l'opéra, leur signification proprement aristocratique, pour ne désigner qu'une valeur morale abstraite, détachée de sa substance sociale. C'est au contraire un des ressorts de la tension dramatique bernanosienne: Blanche doit retrouver son honneur parce qu'elle est d'une grande famille. Dans la scène finale, c'est son honneur aristocratique, transfiguré par la grâce, que Blanche retrouve. Elle devient elle-même, sainte et noble, quand, dans l'opéra de Poulenc, elle obtient la grâce du courage sans que ses origines sociales ne se trouvent dépassées ou transfigurées. Quant à Constance, si elle est sans contestation possible dans l'opéra une sainte joyeuse, à la manière d'un saint François évoquant "notre sœur la Mort corporelle », elle était chez Bernanos le fruit d'une éducation aristocratique ne prenant pas la mort au sérieux ${ }^{11}$.

Contrairement à Poulenc, Bernanos est sensible à la grandeur d'une aristocratie qui se loge au point même où elle se montre d'une faiblesse apparemment dégradante. On ne sait si l'on doit rire ou admirer ces condamnés à mort allant à l'échafaud au milieu d'une partie de cartes et avec une exquise politesse ${ }^{12}$. Corrélativement, la bourgeoisie de madame Lidoine prend un relief particulier dans les Dialogues selon Bernanos : elle compte ses filles comme on compte ses sous, telle une bonne mère " selon la nature ", mais aussi comme une avaricieuse. C'est au moment où elle veut tenir tous ses comptes

\footnotetext{
${ }^{10} \mathrm{Ce}$ que bien des commentateurs affirment comme étant le propre de Bernanos est plutôt le symptôme de l'influence de l'opéra de Poulenc sur la lecture du texte de Bernanos. Par exemple Michel EsTÈvE, « Métamorphose d'un thème littéraire. À propos des Dialogues des carmélites », Études bernanosiennes, $\mathrm{n}^{\circ} 1,1960$, p. $45-75$ (270-298).

${ }^{11}$ Poulenc la nomme la «soubrette de Dieu », ce qui ne convient nullement au personnage originel de Bernanos. Francis Poulenc, lettre du 16 octobre 1961, Cahiers de l'Herne consacré à Bernanos, Paris, 1962, p. 160. Le traitement musical souligne lourdement cet infléchissement: alors que dans le texte de Bernanos, sœur Constance fait une prophétie sans y penser (ou les uns à la place des autres, qui sait ?), tout indique dans l'accompagnement orchestral qu'elle a don de connaissance. Le forte soudain, le changement de pédale, et le passage à la franche tonalité de la après le chromatisme et les modulations jouant sur l'ambiguïté majeur/mineur des mesures précédentes: la musique ôte toute futilité aristocratique aux paroles de sœur Constance. Francis POULENC, Dialogues of the Carmelites [Dialogues des Carmélites], partition pour piano, Milan, Ricordi, 1959, $2^{\mathrm{e}}$ acte, interlude I, 15, p. 108.

${ }^{12}$ L'inspiration de Bernanos ici est peut-être Hippolyte TAINE, Les Origines de la France contemporaine. L’Ancien régime, Paris, Laffont, 1986, p. 128.
} 
en règle vis-à-vis de Dieu, prenant sur elle la dette du martyre pour protéger son bien, qu'elle doit tout donner, elle-même et ses filles. Dans l'opéra de Poulenc en revanche, la seconde prieure n'est plus que le contrepoint positif d'un personnage que le compositeur maltraite beaucoup : celui de mère Marie de l'Incarnation. Supprimant la question de l'honneur aristocratique, cette dernière n'est plus qu'une religieuse dure et téméraire, quand madame Lidoine est toute douceur pour ses filles.

Les coupes de Poulenc ne réduisent donc pas le texte de Bernanos à sa substantifique moelle: le thème de l'honneur, traité dans d'autres œuvres de Bernanos ${ }^{13}$, n'est nullement anecdotique. Bien des commentateurs du texte bernanosien transfèrent sur lui la signification propre au livret de Poulenc.

\section{Le sujet trouble de l'énonciation prophétique dans l'opéra}

Stanley Cavell fait ainsi du fonctionnement de l'opéra un milieu entre le fonctionnement représentationnel du théâtre, où nous regardons d'abord un personnage, et l'image cinématographique, où nous regardons d'abord un acteur récapitulant tous les rôles qu'il a portés.

Dans l'opéra, il semble impossible de décider [...] quel est l'accent placé relativement sur le chanteur et sur le rôle, voire son importance, n'était le fait de la nouvelle conception que cela introduit du rapport entre voix et corps, rapport où ce n'est pas tel acteur et tel personnage qui sont incarnés l'un dans l'autre mais où cette voix est située dans - on pourrait dire désincarnée dans - cette figure, ce double, cette personne, ce masque, ce chanteur, dont la voix, pour l'essentiel, n'est pas affectée par le rôle ${ }^{14}$.

Entre la représentation théâtrale où l'acteur disparaît derrière le personnage ${ }^{15}$, et l'incarnation par l'image filmique où le personnage devient le prétexte d'un acteur, l'opéra met à égalité le chanteur et le personnage, rendant indécidable la question de savoir qui s'exprime sur scène. En assistant à un opéra, nous sommes tout autant intéressés par le destin d'un personnage que par le chanteur. Cette égalité du personnage et de l'interprète, typique de l'opéra selon Stanley Cavell, n'est ni une représentation (comme dans le cas du théâtre) ni une incarnation (comme dans le cas du cinéma), mais plutôt une communion entre des entités distinctes. Le cas des Dialogues des carmélites est alors particulièrement intéressant, car cette communion des personae, inhérente au genre de l'opéra, rencontre le thème théologique de la Communion des Saints au cœur du livret de Bernanos et Poulenc.

Plus qu'une représentation, Dialogues des carmélites met ainsi en place une réactualisation du martyre des carmélites. Pour comprendre ce phénomène, les

\footnotetext{
${ }^{13}$ Michel EsTÈve, « Métamorphose d'un thème littéraire », op. cit., p. 57.

${ }^{14}$ Stanley CAVELL, Un ton pour la philosophie. Moments d'une autobiographie [1994], Paris, Bayard, 2003, p. 195.

${ }^{15}$ On pourrait objecter à Stanley Cavell qu'une même égalité entre le personnage et l'acteur de théâtre se produit. Songeons au cas paradigmatique de Sarah Bernhardt. Ce serait, selon Cavell, passer à côté des spécificités de chaque dispositif. La caméra saisissant le réel selon Cavell, il est normal que le spectateur de cinéma regarde et s'intéresse d'abord à l'acteur, quelle que soit la trame scénaristique qui justifie son apparition sur la pellicule. Le théâtre ne saisit pas le réel, mais nous en abstrait pour représenter quelque chose. Dans cette perspective, un acteur, fût-il un «monstre sacré », est admiré dans sa capacité à se cacher et à se transformer en des rôles écrasants (Phèdre, Médée, Cyrano, etc.), quand on admire une star de cinéma toujours identique à elle-même, non au sens où elle n'évoluerait pas, mais au sens où c'est l'acteur lui-même qui change. L'effet d'incarnation de l'opéra est lié, selon Cavell, à l'égalité entre le personnage et le chanteur. Aucun ne cache l'autre.
} 
analyses de Carolyn Abbate sur les difficultés d'une narrativité musicale sont précieuses. Dans Voix hors-chant ${ }^{16}$, la musicologue soutient que la musique est capable d'une narration qui lui est propre, mais de manière beaucoup plus rare que ne le sousentendent les métaphores habituelles de l'analyse musicale. En effet, le récit n'est pas seulement une séquence événementielle, mais la narration distanciée d'une telle séquence par un énonciateur. Pour qu'il y ait récit musical, il faut donc l'avénement d'une voix musicale à des moments singuliers, et non un vaste tapis sonore redoublant les péripéties physiques et psychologiques d'un livret ou d'un programme. Carolyn Abbate oppose ainsi la conception mimétique de la musique à la narrativité musicale : un récit musical ne peut naître qu'à la condition que cesse le mime musical de l'action ${ }^{17}$. La musique ne produit habituellement pas une narration distanciée, mais au contraire redouble l'action par des événements sonores qui ont effectivement lieu présentement, contrairement aux événements qui ne sont qu'imités par le livret et les chanteurs en action ${ }^{18}$. En tant que théâtre, l'opéra est bien une représentation, mais en tant qu'œuvre musicale, l'opéra est une séquence d'événements sonores. Tout opéra est donc en même temps, sous sa modalité théâtrale, une action représentée, et sous sa modalité musicale, une action qui a effectivement lieu. Il n'en va pas de même au théâtre, où tout ce qui a lieu sur scène doit représenter l'action imitée.

Pour distinguer le mime musical de la narration musicale proprement dite, Carolyn Abbate traite de la chanson narrative dans l'opéra du XIX ${ }^{\mathrm{e}}$ siècle, en s'appuyant sur l'exemple de la ballade de Raimbaut dans Robert le Diable ${ }^{19}$. Dans une chanson narrative, la « petite histoire » résume et annonce la " grande histoire », transformant le récit en prophétie et en mythe ${ }^{20}$. Abbate attribue ce phénomène à l'organisation strophique de la ballade narrative, la «structure répétitive du chant» troublant le « déroulement continu » du récit ${ }^{21}$.

Abbate ne semble toutefois pas remarquer ce qui peut opposer la narration d'une histoire au mythe. Dans un cas, il s'agit de raconter une séquence événementielle passée et close, à laquelle on se rapporte de manière distanciée. La vision du temps présupposée par un tel récit est une ligne continue et irréversible. Au contraire, le régime d'historicité du mythe est celui d'un passé qui est toujours présent : raconter un mythe, ce n'est pas se référer à un passé révolu, mais convoquer une structure événementielle actuelle. Dans cette perspective, on comprend que l'opéra, qui redouble l'action représentée par une séquence événementielle sonore effective, tende à transformer la narration en mythe, et privilégie la forme de la ballade strophique. À l'inverse d'Abbate, nous dirions donc plutôt, en nous appuyant sur ses propres analyses, que la ballade introduite dans l'opéra est un exemple d'échec de la narration musicale à proprement parler, plutôt qu'un exemple paradigmatique de voix récitante.

Dans Dialogues des carmélites, on observe ce phénomène dans le premier tableau, lorsque le marquis de la Force raconte les événements ayant donné lieu à la mort de son

\footnotetext{
${ }^{16}$ Carolyn AbBate, Voix hors-chant. Opéra et récit musical au XIX siècle [1991], Paris, Van Dieren éditeur, 2016.

${ }^{17}$ Carolyn ABBATE, Voix hors-chant, op. cit., p. 45-46.

${ }^{18} \mathrm{La}$ thèse de Carolyn Abbate s'oppose ainsi totalement à l'idée selon laquelle l'opéra serait une épopée, où la musique tiendrait le rôle de récitant, comme le soutient notamment Mauro CALCAGNO, From madrigal to opera: Monteverdi's staging of the self, Berkeley Los Angeles London, University of California Press, 2012.

${ }^{19}$ Carolyn ABBATE, Voix hors-chant, op . cit., p. 110 et $s q$.

${ }^{20}$ Ibid., p. 115.

${ }^{21}$ Ibid., p. 103.
} 
épouse $^{22}$. Il ne s'agit pas d'une chanson narrative telle que l'étudie Abbate, mais on a clairement affaire à un récit dans la trame actionnelle ${ }^{23}$. Dans ce passage, le traitement musical est strictement mimétique : il redouble le récit du marquis, en évoquant le fouet des cochers, l'angoisse de la marquise poussant le verrou et les pas de la foule en panique. Bien que le marquis renvoie expressément à des événements passés ayant eu lieu à une date précise, la musique de Poulenc ne fait pas entendre ici une "voix » propre racontant son propre récit. Elle ne fait qu'illustrer et appuyer le récit du marquis. Mais ce faisant, la musique n'est pas un lointain auquel le discours renvoie: elle réactive les événements, les rendant présents, transformant le récit en hypotypose hallucinatoire, puis en prophétie dont l'actualité demeure confuse, comme toute prophétie biblique. L'énonciation chantante commune au personnage et au chanteur devient ainsi une énonciation prophétique, valant comme telle non seulement à l'intérieur de l'histoire, mais pour le public. Le martyre des carmélites de Compiègne est non seulement prophétisé à l'intérieur de l'histoire, mais prophétisé comme en voie de réactualisation dans l'opéra.

\section{La musique prophétesse}

La musique fait-elle entendre sa propre voix dans les Dialogues des carmélites pour raconter un récit qui lui serait propre ? Si l'on s'appuie sur la cartographie des thèmes musicaux de l'opéra effectuée par Gisèle Philips ${ }^{24}$, la question ne se pose réellement qu'à propos de la figure mélodique de la mort, reprise du Stabat mater de 1951 (montée d'une tierce mineure suivie d'une sixte, selon le rythme régulier de croches ininterrompues).

\section{[Illustration 1]}

Gisèle Philips parle en général à propos des Dialogues des carmélites de leitmotive, ce qui convient pour décrire la manière dont un sens est tissé par la répétition et l'entrelacement de différents thèmes peu à peu reconnaissables par l'auditeur. Toutefois, la référence wagnérienne implicite contrevient trop à la «poétique du différencié » de Poulenc, et son refus de la fusion wagnérienne ${ }^{25}$. Le terme de motif ${ }^{26}$ pourrait convenir, en ce qu'il fait référence à une cellule mélodique clairement identifiable, mais il nous semble difficile d'utiliser le terme de motif sans se référer implicitement à un développement motivique qui ne se déploie jamais. La figure connaît des variations, sans pour autant que la cellule mélodique se transforme en thème, ou se déploie et se métamorphose au fur et à mesure de l'opéra. Nous userons donc de

\footnotetext{
${ }^{22}$ Francis PoulENC, Dialogues of the Carmelites, op. cit., $1^{\mathrm{er}}$ tableau, acte I, 9-10, p. 8-10.

${ }^{23}$ Dans l'édition Béguin, les événements ne sont pas racontés par le marquis, mais présentés dans les " didascalies ".

${ }^{24}$ Gisèle PhILIPS, Essai sur l'oeuvre lyrique de Francis Poulenc, mémoire soutenu au Conservatoire de Paris en 1958, consultable à la BNF cote VMB-1169.

${ }^{25}$ Hervé LACOMBE, «L'idée et la façon : composer selon Francis Poulenc », Lucie KaYAS et Hervé Lacombe (éd.), Du langage au style. Singularités de Francis Poulenc, Paris, Société française de musicologie, 2016, p. 20.

${ }^{26}$ Le terme de «motif» est utilisé dans ce contexte par Markus SCHNEIDER, «La mère des douleurs dansante: une analyse du Stabat mater de Francis Poulenc », Du langage au style. Singularités de Francis Poulenc, op . cit., p. 245-260.
} 
l'expression neutre de figure mélodique, qui a l'avantage d'évoquer l'idée de « configuration au Christ » et le figurisme paulinien ${ }^{27}$.

Contrairement au thème de la force par exemple, qui apparaît dès les premières mesures orchestrales, la figure mélodique de la mort ne se contente pas de mimer et redoubler l'action dramatique : lorsqu'elle apparaît, la musique fait entendre une voix qui lui est propre. La figure mélodique commente l'action et en fait un récit s'écartant de la vision qu'en ont les personnages. S'agissant d'une figure mélodique importée d'une autre œuvre de Poulenc, on peut toutefois se demander si l'on entend la voix de la musique même ou du compositeur ${ }^{28}$. En l'espèce, sans considérer que la voix de la musique soit nécessairement la voix du compositeur assumant la fonction de narrateur ou de récitant, il est incontestable que la capacité narrative de la musique est instaurée par Poulenc par l'autocitation, et donc par une confusion volontaire entre la voix de la musique et l'énonciation auctoriale.

La figure mélodique de la mort apparaît dans l'introduction orchestrale du $4^{\mathrm{e}}$ tableau, consacré à la mort de madame de Croissy ${ }^{29}$.

\section{[Illustration 2]}

Il faut d'abord remarquer qu'elle n'est pas présente dans le $1^{\text {er }}$ tableau, lors du récit de la mort de la marquise de la Force. La figure mélodique de la mort n'est donc pas un thème mimétique d'usage systématique, simple décalque de l'évocation de la mort dans la sphère langagière. La succession d'une tierce mineure et d'une sixte ne pourrait toutefois d'elle-même constituer un récit ou un commentaire distancié si elle n'était pas la citation du Stabat mater de 1951. Poulenc fait entendre la musique comme étant sa propre voix, pour raconter à l'auditeur un récit qui diverge en partie de ce qu'il a sous les yeux : nous n'assistons pas à l'agonie d'une religieuse qui renie toute son existence en criant sa peur animale de mourir, mais mystiquement à la mort du Christ. La voix du compositeur autocitant sa propre musique raconte la scène en faisant un parallèle explicite : alors que dans le Stabat mater, les voix du chœur racontent comment la Mère se tient debout au pied de la Croix en communion avec le Fils, la voix du compositeur dans les Dialogues raconte comment des femmes se tiennent debout aux côtés d'une agonisante. La voix du compositeur, par l'usage de l'autocitation, raconte la transfiguration de l'empathie émotionnelle en une communion avec une figure christique. En ce sens, le spectateur entend bien un récit proprement musical, dont l'énonciation a une source auctoriale, et qui raconte l'action de manière singulière.

Comme dans la ballade narrative décrite par Carolyn Abbate, la répétition inhérente à l'usage de figures mélodiques caractéristiques tend à modifier la temporalité du récit pour en faire un récit mythique ou une énonciation prophétique. En l'espèce, nous avons clairement affaire à la seconde situation. L'énonciation prophétique est ici d'abord celle de la voix musicale, dont la distance par rapport au personnage l'autorise à indiquer une configuration au Christ. Ainsi, la figure mélodique réapparaît au moment où madame de Croissy décrit sa vision apocalyptique d'une chapelle « vide et profanée ${ }^{30} »$. Notons que cette vision n'est pas un récit comprenant un début, un milieu et une fin (contrairement

\footnotetext{
${ }^{27}$ Erich AUERBACH, Figura : la loi juive et la promesse chrétienne [1944], Paris, Macula, 2017.

${ }^{28}$ Edward Cone, The Composer's voice, Berkeley-Los Angeles, University of California press, 1974.

${ }^{29}$ Francis PoulEnC, Dialogues of the carmelites, op. cit., $4^{\mathrm{e}}$ tableau, acte I, 76, p. 65.

${ }^{30}$ Francis PoulENC, Dialogues of the carmélites, op. cit., $4^{\mathrm{e}}$ tableau, acte I, 114-115, p. 91-92.
} 
à la narration initiale du marquis de la Force). C'est par la voix de la musique que la vision d'un lieu sacré dévasté acquière pour l'auditeur le statut de récit prophétique.

Quand le simple récit narre une séquence d'événements passés, et quand le mythe décrit une structure du monde toujours actuelle, la prophétie raconte des événements invisiblement en cours de réalisation, inachevés, dont le terme sera l'accomplissement. Alors la prophétie disparaît et se meut en récit véritable (ayant un début, un milieu et une fin) d'une séquence événementielle passée. La mort de madame de Croissy fait cesser brutalement l'énonciation musicale, soulignant l'inachèvement du récit prophétique.

\section{[Illustration 3]}

La figure mélodique est brisée de manière inattendue : on retrouve la montée d'une tierce mineure (mi-sol), qui ne s'achève cependant pas sur un mi bécarre comme dans la mesure précédente, mais sur un accord de septième du $7^{\mathrm{e}}$ degré ${ }^{31}$, et dissonant (avec un fa bécarre s'ajoutant accidentellement). Les derniers mots de la prieure (peur de la mort) sont posés sur un intervalle de sixte qui n'est pas précédé d'une tierce mineure. On a donc la figure mélodique de la mort décalée et interrompue ${ }^{32}$. Or l'inachèvement de la narration va de pair avec l'énonciation prophétique. Dans la tradition biblique, la prophétie n'est pas seulement une prévision. Son existence participe de ce qui est annoncé, soit qu'elle invite au repentir et à la conversion, soit que les conséquences de la prophétie sur le prophète commencent à réaliser ce qui est proféré. La prophétie est donc à la fois récit d'un événement à venir, et participation à cet événement. Dans une perspective chrétienne, toute prophétie s'accomplit dans le Christ. Jusqu'à son dernier Avènement, elle conserve une actualité prophétique.

\section{La construction du finale comme réactualisation quasi-eucharistique}

L'utilisation de ce thème récurrent ${ }^{33}$ et reconnaissable transforme la conclusion de l'opéra en achèvement et accomplissement de l'énonciation musicale prophétique, commencée dans le $4^{\mathrm{e}}$ tableau de l'opéra. Le récit est enfin complet, ce qui termine de dissoudre la représentation théâtrale pour produire un effet de réel saisissant. Sur le plan musical, c'est la première fois que la figure mélodique de la mort se déploie sur une aussi longue période, sans pour autant qu'elle ne soit développée ou variée (hormis les mesures de modulation). Étêtée de sa tierce finale, la figure mélodique de base n'occupe plus qu'une mesure. Alors que sa version précédente dépassait les limites de la mesure et demeurait ouverte, cette version abrégée est l'énonciation d'une clôture : l'énonciation prophétique s'accomplit, et s'abolit en tant qu'énonciation. Mimant rythmiquement (quasi métronomiquement) la marche à l'échafaud, la musique abandonne sa voix narrative pour redevenir mimésis de l'action. Le récit n'est plus : ne reste que ce qui s'est réellement passé. Toutefois, parce que les prophéties ne furent pas seulement énoncées à l'intérieur de l'histoire racontée, mais l'ont été également pour le

\footnotetext{
${ }^{31}$ Dans la scène finale également, le premier coup de guillotine tombera sur la sensible.

${ }^{32}$ Sur la place des ruptures dans le style musical de Poulenc, Hervé LACOMBE, «L'idée et la façon : composer selon Francis Poulenc », Lucie KAYAS et Hervé LACOMBE (éd.), Du langage au style, singularités de Francis Poulenc, , p. 11-46.

${ }^{33}$ Il réapparaît tout au long de l'opéra, notamment lors de la prophétie de sœur Constance (Pensez à la mort de notre chère Mère, Scur Blanche, Acte II, interlude I, 15) et lors de l'acte de condamnation (acte III, scène III, 49).
} 
public dans des actes d'énonciation chantant (émanant à la fois des personnage et des chanteurs) et par la musique elle-même lorsqu'elle prend la figure de la voix du compositeur, l'accomplissement de la prophétie ne se présente pas seulement comme ayant eu lieu. Elle est en train d'advenir dans l'expérience auditive du spectateur : la musique est d'abord une séquence d'événements sonores présents.

Ce passage de la représentation lyrique à l'actualisation quasi-eucharistique est accentué par le changement de statut du chant ${ }^{34}$ : lorsque le Salve Regina commence à retentir, le spectateur sait qu'il ne doit pas interpréter ce chant comme de la parole dans le médium lyrique. Les carmélites chantent sur scène, car elles chantent réellement. La rupture du médium lyrique produit donc un effet de réel saisissant. Il est par ailleurs évident qu'en 1957, n'importe quel spectateur des Dialogues des Carmélites (a fortiori à Milan) connaît par cœur le Salve Regina, et chante donc intérieurement en même temps que les chanteuses. Le dispositif crée ainsi le sentiment d'une communion avec les martyres, reproduisant la communion décrite dans le Stabat mater.

Une prophétie est toujours ambiguë, et son accomplissement prévisible quoique surprenant. Le positionnement des coups de guillotine dans la scène finale permet à Poulenc de traduire ce phénomène musicalement, tant sur le plan rythmique, harmonique et mélodique ${ }^{35}$. D'un point de vue rythmique, le couperet ne tombe jamais à contretemps, presque toujours sur le premier temps d'une mesure, et une seule fois au commencement d'une formule rythmique. Si l'on considère le texte chanté, le couperet intervient alternativement en fin de mot ou au milieu d'un mot, une seule fois au début d'une phrase, et presque toujours au milieu d'une formule mélodique qu'il n'interrompt pas, hormis le premier coup de guillotine, qui intervient sur la sensible, empêchant la résolution sur la tonique et entraînant une modulation à la quinte. Les autres modulations ne seront pas enclenchées par la guillotine, donnant le sentiment, après l'étonnement de la première exécution, d'une acceptation. Il en va de même de l'impressionnant crescendo, venant contrebalancer l'amoindrissement du nombre de chanteuses, évoquant le courage collectif d'une communauté chantant à l'unisson (ou éventuellement à l'octave). Lorsque le crescendo ne suffit plus, l'unisson est abandonné, faisant entendre les voix uniques de sœur Constance, sœur Mathilde et mère Jeanne, la singularité des sacrifiées apparaissant de manière bouleversante après l'unisson évoquant la communion du Carmel.

Poulenc a effectué un travail conséquent sur le texte même de Bernanos, qui en changea considérablement le sens. Au-delà de ces infléchissements strictement littéraires, les Dialogues des carmélites sont remarquables en ce que le compositeur a utilisé les ambiguïtés du dispositif opératique pour traiter le thème même du livret. L'ambiguïté énonciative de la voix lyrique, décrite par Stanley Cavell, fait sortir la prophétie du cadre intra-diégétique. Les difficultés d'une narration musicale, soulignées par Carolyn Abbate, sont dépassées en la construction d'une prophétie musicale en voie de réalisation au cours de l'opéra. Dans cette perspective, il y avait bien une affinité générique de l'opéra avec le traitement bernanosien de la Communion des Saints. D'une manière inaccessible pour le théâtre ou le cinéma, l'opéra permet la mise en place d'un

\footnotetext{
${ }^{34}$ Violaine ANGER, «Du silence au chant : l'imaginaire de la parole dans les Dialogues des carmélites », Lucie Kayas et Hervé Lacombe (éd.), Du Langage au style. Singularités de Francis Poulenc, Paris, Société française de musicologie, 2016, p. 225-234.

${ }^{35}$ Selon la remarque de Violaine Anger dans l'article précédemment cité, bien qu'elle ne procède pas à 1'analyse.
} 
dispositif où le destinataire ultime de la prophétie, et le bénéficiaire de la grâce du martyre des carmélites, est le public. 